\title{
O‘ZBEK VA TURK TILSHUNOSLIGIDA FE'L MAYLLARINI TASNIFLASH MEZONLARI
}

Zilola XUDAYBERGENOVA *

\begin{abstract}
Annotatsiya
O'zbek va turk tillarida fe'l so'z turkumining asosiy kategoriyalaridan bir mayl kategoriyasi hisoblanadi. Maqolada bu tillardagi so'zlovchining obyektiv borliqqa bo'lgan modal munosabatini ifoda etuvchi fe'l shakllari - mayllar o'zaro qiyoslab tadqiq etish maqsad qilingan. Zero, mazkur masala maxsus tadqiqot obyekti bo'lmagan. Har ikki til uchun umumiy bo'lgan fe'l mayllaridan tashqari, o'zbek va turk tillarida o'ziga xos spesifik bo'lgan, qiyoslanayotgan ikkinchi tilda mavjud bo'Imagan mayl ko'rsatkichlari bor. O'zbek va turk tilshunosligida fe'l mayllarini tasniflash va o'rganishda farqli yondashuvlar mavjud. Shu bilan birga turk tilshunosligida ham, o'zbek tilshunosligida ham turli ilmiy manbalarda fe'l mayllarining turlicha tasniflangani kuzatiladi. Maqolada o'zbek va turk tilshunosligida fe' 1 mayllarining tasniflaridagi mushtarakliklar va farqlar tahlilga tortiladi. O‘zbek va turk tilshunosligida xabar maylining boshqa fe'l mayllariga qarama-qarshi qo'yilgani qayd etiladi. Natijada qiyoslanayotgan tillardagi mayl va zamon kategoriyalarining o'zaro munosabati, ularning turlari, tuzilish tiplari, soni xususidagi fikr-mulohazalarida bir xillik yo'qligi, hattoki, ayni bir fe'l shakli uchun butunlay farqli qarash bayon qilgani e'tirof etiladi. Misollar tahlili natijasida fe'l mayllaridagi obyektivlik va subyektivlik darajalarini mayllar va zamon shakllarini farqlash mezoni sifatida qo"llash tasviya etiladi.
\end{abstract}

Kalit So'zlar: O‘zbek Tili, Turk Tili, Morfologiya, Mayl, Mayl Mezonlari

\section{ÖZBEK TÜRKÇESİ VE TÜRKIYYE TÜRKÇESİ DILLBILIMIINDE FIIIL KİPLERININN SINIFLANDIRMA KRITERLERI}

Özet

Özbek Türkçesi ve Türkiye Türkçesinde fiilin ana kategorilerinden biri fill kipleridir. Bu makalede Özbek Türkçesi ve Türkiye Türkçesi ana konuşmacıların nesnel varlığa yönelik modal tutumunu ifade eden fiil şekillerinin karşılaştırmalı olarak incelenmesini amaçlamaktadır. Çünkü bu konu şimdiye dek özel bir araştırma konusu olmamıştır. Her iki dil için de ortak olan fiil kipleriyle aynı zamanda karşılaştırılan ikinci dilde bulunmayan belirli kipler mevcuttur. Özbek Türkçesi ve Türkiye Türkçesi dilbiliminde fiil kiplerinin sınıflandırılması ve incelenmesi için farklı yaklaşımlar vardır. Aynı zamanda, Türkiye Türkçesi dilbiliminde de, Özbek Türkçesi dilbiliminde de fiillerin kiplerinin türlü bilimselkaynaklarda farklı sınıflandırıldığı görülür. Makalede, Özbek Türkçesi ve Türkiye Türkçesi dilbilimindeki fiillerin kipleri arasındaki ortak ve özellikler incelemiştir. Özbek Türkçesi ve Türkiye Türkçesi dilbiliminde haber kipinin diğer kiplere konulduğu belirtilmektedir. Sonuç olarak, kıyaslanan dillerdeki kip ve zaman kategorilerinin etkileşimi, onların tasnifi, yapıları ve sayıları konusunda oy birliğinin olmadığı, hatta ki, aynı bir fiil formu için tamamen farklı yorumların öne sürüldüğü anlaşılır. Örneklerin analizi sonucu olarak, nesnelliğin ve öznelliğin derecelerinin fiil kiplerinin ve zaman şekillerinin sınıflandırılması için bir kriter olarak kullanılması önerilmiştir.

Anahtar Kelimeler: Özbekçe, Türkçe, Morfoloji, Kip, Kiplerin Sınıflandırma Kriterleri

\footnotetext{
* Prof. Dr., Bartın Üniversitesi Edebiyat Fakültesi Çağdaş Türk Lehçeleri ve Edebiyatları Bölümü, el-mek: aloliz74@mail.ru, zkhudaybergenova@bartin.edu.tr
}

(D) ORCID ID: https://orcid.org/0000-0003-1021-3053 


\title{
CRITERIA FOR DEFINING MOODS OF THE VERB IN UZBEK AND TURKISH LINGUISTICS
}

\begin{abstract}
In the Uzbek and Turkish languages, moods of the verb is one of the main categories. The purpose of the article is a comparative study of the mood of verbs - verb forms, which is expressing the modal attitude of the speakers of these languages to objective reality. After all, this issue was not the subject of a separate study. The verb moods in the compared languages have common and similar grammatical affixes, however, there are also verb moods that do not exist in a comparable second language. There are different approaches to the classification and study of verb moods in Uzbek and Turkish linguistics. At the same time, various scientific sources describe various classifications of moods of verbs in Turkish linguistics and Uzbek linguistics. The article analyzes the differences and similarities between the moods of verbs and their interpretations in Uzbek and Turkish linguistics. The article notes that in Uzbek and Turkish linguistics, the indicative mood contradicts other moods of verbs. As a result, it is recognized that in the languages being compared there is no single approach to the relationship between the category of tenses and the mood of verbs, their classification and types, they even express completely different opinions about the same form of the verb. As a result of the analysis of examples, it is proposed to use the degrees of objectivity and subjectivity as criteria for differentiating the moods of verbs and tenses.
\end{abstract}

Key Words: Uzbek, Turkish, Morphology, Mood, Mood's Criteria

\section{Kirish}

Tilning qudrati shundaki, u hayotda ro'y berayotgan o'zgarishlarni o'zida gavdalantirib, tildagi ma'no nozikligini, ko'p qirralilikni ko'rsatadi, ta'kidlaydi. Shubhasiz, bu hodisalarning tildagi ifodasi inson tafakkuridagi o'ziga xoslikka bog'liq. Avval ta'kidlanganidek, harakat, zamon, makon, o'zgarish obyektiv va mutlaq bo'lgani holda uning kishi ongidagi in'ikosi subyektiv va nisbiydir. Nutqda ham, demak, nisbiylik, subyektivlik ifodalanadi. Tildagi modallik bildiruvchi vositalarning miqdori oz emas. Mayl kategoriyasi nomini oluvchi affikslarning mazmun tarkibidagi subyektivlik va uning ahamiyati, afsuski, mukammal o'rganilmagan. Ammo mazkur hukmdan biron-bir mayl shaklidagi fe'lda obyektiv voqe'lik qayd etilmaydi, degan xulosa kelib chiqmaydi. Faqat obyektivlik subyektivlik qobig'iga o'rab taqdim etiladi, xolos.

Voqe'likning konstatatsiyasi hodisaning uch zamondan birida amalga oshirilishini ko'zda tutadi. Ya'ni shaxs harakat, holatning o'tgan, hozirgi, kelasi zamonlardan biriga taalluqli ekanini ma'lum qiladi. Biroq subyektivlikdan xoli obyektivlik, real voqe'likka bog'liq bo'lmagan subyektivlik mavjud emas. Shu sababli tildagi mayl va zamon hodisasini tadqiq etish orasidagi uzviy aloqadorlikni hisobga olishni taqozo etadi.

\section{O'zbek tilidagi mayl kategoriyasining tadqiqi masalasi}

O‘zbek tilshunosligida, shuningdek, boshqa turkiy tillarga oid adabiyotlarda mayl va zamon kategoriyalarining mohiyatini tushunish va ta'riflashda ziddiyat yo'q. Adabiyotlarda ta'kidlanishicha, mayl kategoriyasi harakat yoki holat bilan subyekt orasidagi aloqaning voqe'likka munosabatini ko'rsatadi. Biroq tilshunoslar o'rtasida mayl va zamon orasidagi chegarani belgilash, bu kategoriyalarni ifodalovchi shakllarni tasnif qilish kabi qator masalalarda yakdillik yo'q. Tilshunoslarning bir guruhi ayrim formalarni u yoki bu xususiyatlariga ko'ra mayl kategoriyasiga kiritsalar, boshqalari shu shakllarni bo 'lak jihatlariga asoslanib, zamon kategoriyasi sifatida farqlashadi. 
Adabiyotlarga murojaat qilsak, ko'pchilik ishlarda o'zbek tilidagi fe'l maylining uch turi qayd etilganini ko'ramiz: aniqlik, buyruq-istak hamda shart mayli(G'ulomov, 1964; Tursunov vd, 1992; Rahmatullaev, 1993; Reshetov, 1961).

Ba'zi tadqiqotlarda maylning to' $r t$ turi tilga olinadi. Bunda buyruq va istak mayli ajratib o'rganiladi (Kononov, 1960 : 202). T.Xo'jaev bu to'rt maylga yana ikki mayl turini qo'shadi, ya'ni muallif aytsa ham (aytsa-da) tipidagi shakllarni to'siqsizlik mayli, aytishi kerak shaklidagi qo'shilmalarni vazifa mayli deb o'rganishni tavsiya etadi(1963 : 103). A. Hojievning ishlarida maylning turlari o'zgacha ko'rsatiladi. Tilshunos -moqchi affiksi bilan yasaluvchi yozmoqchi shaklidagi formani alohida mayl turi sifatida farqlaydi hamda uni maqsad mayli deb ataydi. Shuningdek, ishlar edi tipidagi shakllarni yasovchi -ar edi vositasini shartli maylning formanti deb ta'riflaydi. Shunday qilib, A. Hojiev hozirgi o'zbek tilida maylning quyidagi besh turi bor, deb hisoblaydi: buyruq-istak, shart, shartli, maqsad va ijro mayllari(1973 : 115). M.Sodiqova fe'l mayllarini stilistik aspektda tahlil qilish uchun quyidagicha guruhlashtirishni maqcadga muvofiq deb topadi: 1) bayon-darak mayli; 2) buyruq mayli; 3) shart mayli; 4) maqsad mayli; 6) gumon-taxmin mayli(1976 : 10). Tilshunos -(a)r, -mas (-saykin, -mikin) affikslari bilan hosil bo'luvchi fe'llarni taxmin-gumon mayli deb hisoblaydi. Xabar maylini esa bayon-darak mayli deb atashni ma'qul topadi.

Sh.Shukurov esa bu mayllardan faqat uchtasini: buyruq-istak, shart va aniqlik mayllarining mavjudligini tan oladi hamda tilga olingan boshqa mayllarning "ajratilishi isbot talab qiladi, " deya ta'kidlaydi(1976: 7).

Sh.Shukurovning talabi o'rinli bo'lib, haqiqatdan ham, mayl va zamon kategoriyalarini belgilovchi mezonlar ko'rsatilmagan. Mayl turlarni ajratishdagi har xillikning sababi ana shundadir. Bir affiks ba'zan mayl, goxida zamon yasovchi qo'shimcha sifatida qayd etiladiki, rus tilshunos-turkologi N.A.Baskakov buning sababini quyidagicha izohlaydi: Mayl va zamon kategoriyalari genetik jihatdan bir-biriga yaqin bo'lganligi uchun ularning nafaqat semantik, balki morfologik jihatdan xam qat'iy differensatsiyalashmagani ko'rinadi. Mayl va zamon kategoriyalarini farqlashda yuzaga kelgan turli fikr-mulohazalar asosi shunga borib taqaladi. Birinchidan, bir vaqtning o'zida mayl va zamon ma'nolari uchun ikki affiks qator ishlatilmaydi. Ikkinchidan, bir forma bir tomondan mayl ma'nosini ifodalash uchun xizmat qilsa, ikkinchi tomondan ish-harakatning zamonga munosabatini ko'rsatishi mumkin(Baskakov, 1966 : 280). Ya'ni bir affiksda ham mayl, ham zamon ma'nosi o'z ifodasini topadi. kelmoqchi so'zi maqsad mayliga xosligi aniq, lekin bu misolda so'zlovchi so'zlayotgan vaqtda yoki undan so'ng bajarilishi maqsad qilingan harakat anglashiladi, ya'ni hozirgi-kelasi zamon ma'nosi mavjud. Chunki bugun, hozir yoki ertaga kelmoqchi deyish mumkin. Biroq aslo kecha kelmoqchi emas. kelgan so'zi esa xabar maylida bo'lgani holda uzoq o'tmishga oid harakatni bildirib, natija ma'nosini anglatishi ko'p bora tilga olingan.

Bir guruh tilshunoslar xabar mayli sistemasidagi zamon shakllariga batafsil to 'xtalishadi, ammo qayd etilgan xususiyatni hisobga olmay, bo'lak mayllarning zamonga bog'liq shakllarini inkor etishadi. Masalan, maqsad mayli «faqat maqsad, mo'ljal ma'nosini bildirib, zamon ma'nosiga daxli yo'q» (Shoabdurahmonov vd, 1980 : 262). Yoki: «Shart maylining hech qanday zamon ko'rsatkichiga ega bo'lmagan borsam, aytsa tipidagi formasi zamon ma'nosiga ega emas va $u$ shart maylining biron zamon formasi sifatida qatnashmaydi»(Shoabdurahmonov vd, 1980 : 246). A.N.Kononov, N.A.Baskakov, A.Shcherbak, V.V.Reshetov kabi tilshunoslar esa turkiy tillardagi buyruq maylidan boshqa mayllar sistemasida zamon kategoriyasi mavjudligini e’tirof etishadi(Kononov, 1960; Baskakov, 1966; Sherbak, 1962).

Demak, bu tilshunoslarning fikriga ko'ra, xabar mayli boy zamon sistemasiga ega bo'lsa, boshqa mayllar nisbatan kam sonli zamonlar shaklini o'z ichiga oladi. 


\section{Turk tilidagi mayl va zamon kategoriyalarining talqini}

Turk tilidagi mayl va zamon kategoriyalari xususida J.Deni, G.Veyl, I.Vige, A.N.Kononov, A.A.Gordlevskiy, N.K.Dmitriev, X.Javdotzoda, N.N.Janashia, Muharram Ergin, Tahsin Bangi o'g'li, M.Erdem kabi olimlar fikr yuritgan(Deny, 1921; Deny, 1969; Weil, 1917; Viguier, 1790; Gordlevskiy, 1928; Dmitriev, 1960; Grunina, 1961; Kononov, 1966; Genjan T, 1971; Ivanov, 1977; Djanashia, 1981; Ergin, 1993; Bangioglu, 1990; Toparl1, 1991; Erdem, 2016).

Fe'lning bu kategoriyalarini ta'riflashda ba'zi olimlarning nuqtai nazarlari bilan o'zbek tilidagi ayni hodisalar tavsifi o'rtasida tafovut yo'q. Ammo turk tilshunoslari ushbu tushunchalarga tamomila o'zgacha baho beradilar. Shuningdek, fikrlarining isboti uchun yangi atama ostida yangicha tushunchani iste'molga kiritadilar. Fransuz tilshunoslarining turk tilidagi fe'lning o'ziga xos xususiyatlari xususidagi fikrlari turk tilshunoslarining fikrlaridan deyarli farq qilmaydi. Masalan, J.Deni turk tilidagi fe'l shakllarini sakkiz guruhga ajratadi: tugallangan o'tgan zamon fe'li (geldi), shart mayli (gelse), hozirgi zamon (geliuog), aorist (gelir), o'tgan zamon hikoya shakli (gelmiş), kelasi zamon maqsad fe'li (gelesek), keraklilik shakli (gelmeli), istak shakli (gele). J.Deni idi, imiş, ise fe'l shakllari yordamida fe'lning hikoya, rivoyat, shart murakkab shakllarini yasaladi, deb hisoblaydi. Sakkiz sodda shakl bu vosita yordamida yigirma to'rt fe'l shaklini yuzaga keltiradi. Bu yigirma to'rt shakldan uchtasi qo'llanmaydi (Bu haqda quyida Muharram Erginning ishlarida batafsil to'xtalamiz). Yigirma bitta murakkab va sakkizta sodda fe'l shakli bilan turk tilida 29 fe'l shakli bor. Umuman, turk tilidagi fe'lning 43 zamon va mayl shakli qayd etiladi(Deny, 1969 : 209-211).

Prof. Muharram Ergin zamon va mayl atamasi bilan birgalikda shakl (şekil) terminini qo'llaydi. Bu o'rinda mayl va shakl atamasi deyarli parallel ishlatiladi. M.Ergin bu shakllarni ikkiga ajratadi: xabar mayllari (bildirme kipleri) va reja mayllari (tasarlama kipleri). (Ergin, 1993 : 336).

Xabar mayllari bajarilgan yoki bajariladigan harakatni bildirsa, reja mayllari bajarish rejalashtirilgan ish-harakatni anglatadi. Xabar mayllari harakat-holatning bajarilish vaqtini ifodalashi mumkin. Biroq, olimning fikricha, reja mayllari bu imkoniyatdan mahrum. Xabar mayllari $o^{6} z$ ichida besh zamonga ajraladi: hozirgi-kelasi zamon (genis zaman), hozirgi zamon (şimdiki zaman), noaniq o'tgan zamon (ögrenilen geçmiş zaman), aniq o'tgan zamon (görülen geçmiş zaman), kelasi zamon (gelecek zaman); reja mayllari to' $r$ th xildir: shart (şart), istak (istek), keraklilik (gereklilik), buyruq (yetir). Demak, Muharram Erginning xulosasiga ko'ra turk tilida to'qqiz mayl bor (Ergin, 1993 :337). Xususan, turk olimi fe'llarning qo'shma mayllari (birleşik) borligini e'tirof etadi. Xabar va reja mayllarining imek to'liqsiz fe'lining aniq o'tgan zamon, noaniq o'tgan zamon va shart maylidagi shaklida qo'llanishiga qo'shma maylli tuslanishi deb qaraladi. idi yoki qisqa shakli -di bilan hosil qilinuvchi shakl hikoya (hikaye), imiş (-miş) ishtirokida yasaluvchi forma rivoyat (rivayet), ise (-se) yordamida hosil bo'luvchi forma shart (şart) deb ataladi. Ayni tushuncha Tahsin Bangi o'g'li tomonidan tarz deb nomlanadi. Natijada turk tilida yigirma bitta mayl (tuslanish)borligi ma'lum bo'ladi. O'zbek tilida hozirda bog'lovchi vazifasini bajaruvchi esa so'zi turk tilida to'liqsiz fe'l sifatida qo'llanish imkonini yo'qotmagan, hattoki bu so'zning imkoniyati katta. Shu sababli turk tilidagi qo'shma mayldan so'ng ise (-se/-sa) so'zini keltirish qavatli qo'shma maylni vujudga keltiradi, deyiladi Muharram Erginning tadqiqotida(1993 : 344). Bunday shakl turk tilida ikkitadir. Bugungi kunda shartning kavatli qo'shma mayli yo'q bo'lib, hikoya va rivoyat shakli qavatli qo'shma tuslanish mayliga ega. Masalan, hozirgi-kelasi zamon hikoyasining sharti: geliyorset (geliyor isem). Tahsin Bangi o'g'li bu kabi shakllarga to 'xtalmaydi. Demak, Muharram Erginning ta'kidicha, to'qqizta sodda, yigirma bitta qo'shma va o'n bitta qavatli qo'shma mayl bor.

Turk tilshunosi Tahsin Bangi o'g'li tuslanishli fe'lning asosiy to'rt shakli deb quyidagilarni biladi: zamon, tarz, shaxs, son. Mayl esa quyidagi omillarni talab qiladi: "Zaman 
(varsa tarz) eki ile uzatılmış bır fiilin tekli ve çoklu kişi ekleri almasiyla bır kip meydana gelir' ( Zamon (bor bo'lsa tarz) qo'shimchasini olgan fe'lning shaxs-son affikslarini olishi, tuslanishi natijasida mayl yasaladi).

Tahsin Bangi o'g'li tuslanishli fe'l uchun zamon kategoriyasini asosiy omil deb topadi va mayllar ham aslida zamon bo'limlariga ko'ra nomlanganligi uchun mayl termini o'rnida zamon atamasini qo'llash mumkinligini ta'kidlaydi. Olim $\mathrm{o}^{6} \mathrm{z}$ tadqiqotida ikki xil zamonni farqlaydi(Bangığlu, $1990: 441$ ):

1. Nutq oniga ko'ra belgilanadigan zamonlar — o'tgan, hozirgi, kelasi zamonhisobiga yolg'iz zamon (salt zaman) deyiladi.

2. Harakat nuqtasini nutq onidan, ya'ni hozirgi zamondan boshqa zamon qobig'iga o'tkazish mumkinki, bunday zamon shaklin nisbiy zamon (göreli zaman) hisoblanadi. Masalan, uazıuor fe'liga o'tgan zamon ko'rsatkichi -di qo'shilsa, harakat nuqtasi o'tgan zamonga ko'chadi. uazıyordu shaklida ikki qo'shimcha mavjud bo'lib, birinchi affiks hozyrgi zamonning, ikkinchi affiks o'tgan zamonning ko'rsatkichidir. Bu formadagi -du tarz kategoriyasining formantidir. Tahsin Bangi $\mathrm{o}^{6} \mathrm{~g} \mathrm{~g}^{\prime} \mathrm{li}$ M.Erginning ishida qo'shma mayl sifatida ta'riflangan fe'l shakllarini tarz kategoriyasiga kiritadi.

Tilshunos turk tilidagi quyidagi asosiy zamon va yon zamon shakllarini ko'rsatadi: 1.O'tgan zamon (geçmiş zamap) -di affiksi yordamida yasaladi. 2. Hozirgi zamon (şimdiki zamap) -uor affiksi yordamida yasaladi. 3. Kelasi zamon (gelesek zatap) asak affiksi yordamida yasaladi. 4. Bilvosita o'tgan zamon (dolayıl geçmiş) -miş qo'shimchasi orqali hosil qilinadi. 6. Hozirgi-kelasi zamon (geniş zaman) -r, -ir qo'shimchasi vositasida hosil qilinadi. Bu fe'l shakllaridan ilk uchtasi asosiy zamon, keyingi ikkitasi yon zamondir(Bangığlu, 1990 : 441444).

Shunday qilib, Tahsin Bangi o'g'li xabar mayli tizimidagi to "qqiz zamon vositasi bilan to 'qqiz maylni, to'qkiz zamon va to'rt tarz yordamida hosil bo'ladigan o'ttiz maylni ko'rsatadi.

Turk tilshunosi Vahit Tanening fikriga ko'ra turk tilidagi o'tgan zamon shakllari mohiyat e'tibori bilan o'z ichida ikki qismga ajraladi(Tane, 1994): a) aniq o'tgan zamon (görülen geçmiş zaman); b) o'tgan zamon eshitilganlik fe'li (duyulan geçmiş zaman). U Tahsin Bangi o'g'li tarz, Muharram Ergin qo'shma mayl deb farqlagan shakllarni qo 'shma zamon deb ataydi. Natijada besh sodda, o' $\mathrm{n}$ beshta qo'shma zamonni (jami: yigirma zamon shakli), to'rt sodda va sakkiz qo'shma (o'n ikkita) maylni belgilaydi. V.Tanening tasniflarida mayl va zamon o'rtasidagi bog'liqlik inkor etiladi. Ya'ni zamon shakli maylning, mayl shakli zamonning xususiyatlarini bildirmaydi. Tahir Nejat Genjan fe'llar zamon va ma'nolari jihatdan turli qo'shimchalarni oladiki, ularning har biriga mayl deyiladi, deya e'tirof etadi. Uning ishida fe'lning to'qqizta mayl keltiriladi: -di shaklli o'tgan zamon mayli — aniq o'tgan zamon fe'li, miş li o'tgan zamon mayli - taxminiy o'tgan zamon fe'li, hozirgi zamon mayli, kelasi zamon mayli, hozirgi-kelasi zamon mayli hamda keraklilik, istak, tilak-shart, buyruq mayllari. Bulardan birinchi beshtasi xabar mayllari. keyingi to'rttasi esa istak mayllari hisoblanadi. Xabar mayllarida zamon tushunchasi doimo mavjud bo'lib, istak mayllarida deyarli sezilmaydigan kelasi zamon ma'nosi bor. Shu bois istak mayllari asosiy ma'nolariga ko'ra nomlangandirlar. T.N.Genjan bu har bir maylning shart, rivoyat va hikoya shakllari — qo'shma zamonli fe'l shakllari qo'llanishini qayd etadi(Genjan, 1971 : 233-248). Demak, qayd etilgan avvalgi tadqiqotlardan farqli o'laroq T.N.Genjanning ishida istak mayllarida kelasi zamon tushunchasi mavjudligi e'tirof etiladi. Shunday bo 'lsa-da, bu tilshunos ham zamon va maylni bir tushuncha deb baholaydi hamda turk tilidagi maylni zamondan farqlovchi belgisini ko'rsatmaydi. R.Toparlining ishida ham sodda va qo'shma tuslanishli fe'llar farqlanadi. Sodda affiks vositasida yasaluvchi fe'l shakllariga geldı, gelmiş, geliyor, gelir, gelecek, ge1, gele, gelmeli, gelse tipidagi fe'l formalarini kiritadi. Qo'shma fe'l shakllari esa idi, imiş, ise to 'liqsiz fe'llarini qayd etilgan fe'l shakllariga qo'shish yordamida hosil qilinuvchi formalarni qamrab oladi(Toparl1, 1991 : 52-70). Tilshunos zamon va mayl atamalariga murojaat qilmaydi, u bu 
kategoriyalarni ifodalashda "tuslanish" so 'zidan foydalanadi. Olimning ishida fe'lning to 'qqizta sodda va yigirma bitta qo'shma tuslanishli (ya'ni mayl hamda zamon) shakli ko'rsatiladi.

Vejihe Xatib o'g'lining ishlarida ham tilga olingan turk tilshunoslari fikrlaridan tubdan farqlanuvchi zamon shakllari berilmaydi. Tilshunos fe'lning sodda va murakkab zamon shakllarini ajratadi, xususan qavatli qo'shma zamon kategoriyasini alohida o'rganadi(Hatıboğlu, 1969 : 17; Hatıboğlu, V , 1974 : 59-60). Asosiy tafovut muayyan fe'l shaklini nomlashga borib taqaladi. Masalan, kesin geçmiş zaman - aniq o'tgan zamon, görülen geçmiş zamonko'rilgan o'tgan zamon atamasi bilan berilgan -di shaklli fe'l ushbu tilshunos tadqiqotida belirli geçmiş zaman - aniq o'tgan zamon atamasi bilan tadqiq etiladi.

Turk tilidagi fe'lning xususiyatlarining o'rganishga oid yangi fikrlar M.Erdem tomonidan ilgari surilgan. Tilshunos turk tishunosligidan an'anaviy qarashlardan farqli bo'lgan fe'l tasnifini amalga oshirgan, ya'ni turk tilidagi fe'llarning morfologik, sintaktik va semantik xususiyatlarini alohida o'rganishni taklif etgan (Erdem, 2016 : 185-200.). Biroq bu manbada fe'lning mayl va zamon shakllariga oid mulohazalar bayon etilmagan.

Demak, turk olimlarining fe'lning mayl va zamon kategoriyalari bo'yicha amalga oshirgan tasniflari bir-biridan deyarli tafovutlanmaydi. Asosiy farq fe'l shakllarining nomlanishida ko 'zga tashlanadi.

A.A.Gordlevskiyning tadqiqotidagi mayl tasnifi A.N.Kononov va X.Javdotzodaning tasnifidan faqat bir nuqtaga ko'ra farq qiladi. A.N. Kononov subyektiv modallik tarzida ajratgan formani A.A. Gordlevskiy subyektiv mayl, deb ta'riflagan. N.K. Dmitriev esa turk tilidagi mayl va zamon kategoriyalariga umuman to 'xtalgan bo'lsa-da, bu kategoriyalarni tasnif qilmagan.

Ushbu turkologlar turk tilidagi besh mayl turini tan olishgan: buyruq mayli, keraklik mayli, istak mayli, shart mayli va xabar mayli.

K.M.Lyubimov hozirgi turk tilida oltita mayl borligini e'tirof etadi. Yuqorida tilga olingan mayllar bilan bir qatorda -r va -rdi affiksli fe'l shakllari mavhum mayl formanti sifatida ko'rsatiladi. Bu maylning muvofiq ravishda hozirgi-kelasi va o'tgan zamon (noaniq imperfekt) shakllari ajratiladi(Lyubimov, 1970 : 44). Turk tilidagi fe'lning morfologik belgi-xususiyatlarini o'rgangan gruzin olimi N.N.Janashianing tasnifi A.N.Kononovning tasnifidan deyarli farq qilmaydi. U ham turk tilida ayni besh maylni ajratishning tarafdori(Djanashia, 1981).

N.N.Janashia turk tilidagi fe'lni tadqiq etish borasida yangi kategoriya akt kategoriyasini kiritadi. Bunga sabab turk tilidagi -miş affiksidir. Bu affiks natija ma'nosini ifodalashdan tashqari bilvositalik ma'nosini anglatadi. -miş yordamida hosil qilinadigan murakkab fe'llardagi zamon bu formaning birinchi komponentiga va kontekstdagi umumiy zamon ma'nosiga bog'liq. Masalan, uazıyordu va uazıyormuş formalari o'zaro so'zlovchining nutqiga munosabati nuqtai nazaridan bir xil, ya'ni bu ikki shaklning mayli xabar maylidir. Zamon jihatdan har ikkala so'z ham o'tgan zamonga oid. Mazkur shakllar orasidagi tafovut akt kategoriyasiga asoslanadi. Shu tariqa N.N.Janashia ikki aktni ajratadi: 1) bevosita (ochniy); 2) bilvosita(zaochniy) (Djanashia, 1981).

N.N.Janashia tasnifining yana bir farqli jihat shundaki, tilshunosning shart maylining yangi turini kiritadi: shart I mayli va shart II mayli. Bu guruhlanish shart yasovchi affiksning fe'lning negiziga yoki zamon formasiga qo'shilishiga asoslangan. Shart I mayli bevosita fe'l o'zagiga qo'shiladi: bilse, gelse. Shart II mayli fe'lning ma'lum bir zamon shakliga qo'shiladi: gelecektiyse, gelmiştiyse. A.N.Kononov bu hodisani ise yordamida shakllanadigan shart modalligi deb ta'riflagan. Ayni shakl M.Erginda qavatli qo'shma mayl deb nomlangan.

Demak, turk tilidagi fe'lning mayl va zamon kategoriyalari ham bir qator turk, fransuz, rus, gruzin olimlarining tadqiqot obyekti bo'lgan. Bu yo'sindagi ishlarda ikki farqli yo'nalishni qayd etib o'tish lozim. Fransuz grammatiklari ta'sirida ish olib borgan turk tilshunoslarining fikr, mulohazalari rus, gruzin tilshunoslarining xulosalaridan ko'p jihatdan tafovutlidir. Albatta, 
ayrim fe'l shakllarini nomlashda, tavsiflashda bir xillik, umumiylik bor. Biroq mayllar va zamonlarning miqdorini belgilashda, turlarini ajratishdagi har xillik yaqqol ko'zga tashlanadi.

Turk tilshunoslari, avvalo, mayllarning tuzilishiga ko'ra uch turini ajratishadi:

1. Sodda mayl(kip - mayl, şekil-shakl, çekim-tuslanish atamalari orqali ifodalanadi).

2. Qo'shma mayl (birleşik kip(şekil, çekim).

3. Qavatli qo'shma mayl(katmerli birleşik kip(şekil, çekim).

Rus turkologlarida esa maylning faqat sodda turi farqlanadi. Turk tilshunoslarining fikricha, bir affiks yordamida yasalgan fe'l shakli sodda maylni yuzaga keltiradi. Masalan, quyidagi gap tarkibida ishlatilgan anladım /tushundim so'zi sodda mayl xabar maylining o'tgan zamon shaklidir:

\section{Artık anladım Azize'cigim(H.E.Adıvar).}

\section{Endi tushundim, Azizaginam.}

Rus turkologlari nuqtai nazari ham ayni xulosani qamrab oladi. Lekin turk tilshunoslari ikki formantdan - sodda mayl ko'rsatkichi va to'liqsiz fe'llardan bnrining birikuvi natijasida hosil bo'luvchi fe'l shaklini qo'shma mayl deb hisoblashadi. Masalan, quyidagi misolda keltirilgan -asak idi affiksli fe'l gelecak zamanın hikayesi - kelasi zamonning hikoya shakli sifatida e'tirof etiladi:

Zeyno 'ya bu gece mektup yazacaktı(H.E.Adivar).

Zeynoga bu kecha aniq maktub yozmokchi edi.

N. Mahmudov va E.G.Naskalining turkcha-o'zbekcha tilshunoslik atamalari muqobillariga bag'ishlangan maqolasida gelecek zamanın hikayesi atamasiga kelasi zamon lozim fe'li termini muqobil sifatida beriladi ${ }^{1}$. Bizningcha, mazkur atamaning muqobili to ' $\mathrm{g}$ 'ri tanlanmagan. Chunki -asak idi formantli fe'l kontekstda nutq onidan avval bajarilishi kerak bo'lgan ish-harakatni bildirib, -(i)shi kerak edi formali fe'lga mutanosib ma'no va vazifada inglatilsa ham, bu, mazkur qo'shimchaning yagona spesifik belgisi emas.

Qavatli qo'shma mayl esa «zamon yoki mayl qo'shimchasi+to'liqsiz fe'l+ise to 'liqsiz fe'li» shakliga ega, ya'ni to'liqsiz fe'llardan ikkitasini zamon yoki mayl formantidan so'ng ketma-ket qo'llash vositasida hosil qilinadi:

\section{O tektur uazasakttysa, mutlak gelmeliydi(H.E.Adivar).}

Agar u maktub yozadigan bo 'Isa. albatta kelishi lozim edi.

Bu gapda qatnashgan uazasakttysa fe'li uazmak fe'lidan so'ng -asak kelasi zamon ko'rsatkichi, idi to'liqsiz fe'lining qisqa shakli, ise to'liqsiz fe'lining qisqa shakli birikuvidan yasalgan. Bu fe'l shakllari o'zbek tilidagi perifrastik fe'l formalariga muvofiq.

Qo'shma mayl formasi sifatida ta'riflangan fe'l shakllarini rus turkologlari o'tgan zamonning murakkab (analitik) formalari degan fikrni ilgari surishadi. Qavatli qo'shma mayl shakllari xususida esa rus tilshunoslarining tadqiqotlarida deyarli ma'lumot berilmagan.

Rus, gruzin tilshunoslari turk tilidagi maylning beshta, ba'zan oltita turini ko'rsatishadi. Ularning qayd etishicha, har bir mayl (buyruq maylidan tashqari) o'z zamon shakllariga ega. (Ba'zan buyruq maylining o'tgan va hozirgi zamon shakli ham ajratiladi).

Demak, turk tilida mayl va zamon kategoriyalarining o'zaro munosabati, ularning turlari, tuzilish tiplari, soni xususidagi fikr-mulohazalarida bir xillik yo'q. Hattoki, bir fe'l shakli uchun butunlay farqli qarash bayon qilinadi. Masalan, turk tilshunoslari zamonning murakkab shaklini inkor etib, maylning murakkab shaklini e'tirof etishadi. Rus turkologlari, aksincha, zamonning analitik shakllari mavjudligini qayd etishadi. Shunisi e'tiborliki, rus olimlarining 
ishida turk tilshunoslarining bu mavzudagi tadqiqotlariga deyarli munosabat bildirilmagan. $\mathrm{Bu}$ nuqtai nazarlardagi mazkur tafovut mayl va zamon kategoriyalarining bevosita uzviy aloqada ekanligi amda mayl formalari asosida oz bo'lsa-da, zamon ma'nosining mavjudligi va aksincha, zamon formalari vositasida so'zlovchining munosabati aks etganligi sababli yuzaga kelgan.

Savol tug'iladi: Xo'sh, qo'shma mayl, qavatli qo'shma mayl atamalari o'z oldiga qo'ygan maqsadni oqlaydimi? -yordu, -acaktı tipidagi fe'llarni o'tgan zamon fe'lining murakkab shakli deb hisoblash to'g'rimi yoki ayni fe'l shakllarini qo'shma mayl turini yasaydi, deyish joizmi?

Misollarga murojaat qilamiz:

Teuze, elbise değiştirmek için odasına girmişti, ama kapısını açık bırakmuştı(H.E.Adıvar).

Xola kiyimini almashtirshi uchun xonasiga kirgan, ammo eshigini ochiq qoldirgan edi.

Ushbu gapda ishtirok etgan girmişti- kirgan edi, bırakmıştı - qoldirgan edi so 'zlari -miş o'tgan zamon fe'lini yasovchi formant bilan idi to'liqsiz fe'lining qisqa shakli orqali yasalgan. Turk tilshunoslarining e'tirofiga ko'ra, bu fe'l shakllari qo'shma mayl bo'lib, eshitilgan o'tgan zamonning hikoya shaklidir. Rus turkologlarining fikri bo'yicha xabar mayli o'tgan zamon fe'lining analitik shakllaridan biri. Mayl, ma'lumki, subyektning obyektga modal munosabatini ifodalaydi. Shunday ekan, -mişti affiksli fe'l qo'shma mayl sifatida so'zlovchining munosabatini aks ettirishi lozim edi. Biroq gapdan anglashilgan voqea-hodisaning bayonidan bo'lak boshqa modal munosabat ma'nosi anglashilmaydi. Bu gapda, albatta, so'zlovchining o'tmishga oid voqeaga nisbatan muayyan munosabati aks etgan, ya'ni girmişti - kirgan edi fe'li nisbatan uzoq o'tmishga oid harakatni bildiradi, bırakmıştı - qoldirgan edi fe'li esa harakatning holatga o'tish jarayonini ifodalaydi. Shuningdek, turk tilshunoslari qo'llagan atamadan ham sezilib turibdiki, -miş affiksiga to'liqsiz fe'l qo'shilganda ham (agar u sodda mayl bo'lsa); asl ma'nosi eshitilganlik ma'nosini yo'qotmasligi zarur. Chunki -miş qo'shimchasining vazifalaridan biri shundaki, so'zlovchi bu fe'l shakli vositasida voqeani ko'rmagandek, boshqa manbalardan xabardor bo'lgandek tasvirlaydi:

Hiç gelmemeniz gereken memleketlere düşmüsş̧ünüz (R.H.Karay).

Kelmasligingiz zarur bo 'lgan mamlakatlarga boribsiz.

Masalan, quyidagi misoldagi haber vermeliydim - xabar berishim kerak edi tarkibida kelgan -meli keraklilik mayli qo'shimchasi idi to 'liqsiz fe'lining qisqa shakli bilan ishlatilsa ham birlamchi ma'nosi yo'qolmagan. Ya'ni muayyan ish-harakatni bajarish zaruriyati ma'nosi saqlangan, biroq o'tgan zamon doirasiga ko'chirilgan:

Fakat bir noktayl önceden haber vermeliydim: Prenses azıcık hastadır (R.H.Karay).

Faqat bir narsani avvalroq xabar berishim kerak edi: Malika biroz xasta.

-miş idi shaklli fe'lga e'tibor qilsak, -miş qo'shimchasiga xos tilga olingan belgining saqlanmaganligini, so'zlovchi o'tmishga oid harakat-holatni guvoh sifatida tasvirlashi ma'nosi anglashilishini ko'rish mumkin:

Fakat Yeşilköyde artık kati olarak, Hasanın kalbindeki asrari öğrenmeye karar (H.E.Adlvar).

Faqat Yashil qishloqda endi Hasannning qalbidagi sirlarini aniqlashga qat'iy qaror qilgan edim.

-yor idi shaklli fe'l turk tilshunoslari tomonidan hozirgi zamonning hikoya qo'shma mayli deb nomlanishiga qaramay, so'zlovchining modal munosabatini aks ettirish bu grammatik formaning birlamchi ma'nosi emas. Masalan, quyidagi gapda qatnashgan -yor idi affiksi idi to'liqsiz fe'li ishtirokisiz -uor shaklida ishlatilsa, yagona farq hozirgi zamonga oid ish- 
harakatning o'tmish planiga ko'chirilganligida ekanligi ayon bo'ladi.

Qiyoslang:

Vep yüzümü göktekı bu sıcak noktaya çevirmiş halde yürümeye çalışıyordum (R.H.Karay).

Men yuzimni ko 'kdagi issiq nuqtaga o 'girgan holda yurishga harakat qilayotgan edim (O'.Hoshimov)..

Vep yüzümü göktekl bu sicak noktaya çevirmiş halde yürümeye çalışıyordum(H.E.Adlvar).

Men yuzimni ko 'kdagi issiq nuqtaga o 'girgan holda yurishga harakat qilyapman.

Xuddi shu singari qavatli qo'shma mayl tariqasida farqlangan fe'l shakllarini ham alohida mayl turi deb hisoblash yetarli asoslanmagan. Birinchidan, qavatli qo'shma maylning ko'rsatkichi birgina ise/ esa to'liqsiz fe'lining to 'liq yoki qisqa shakli vositasida yasaladi. Ikkinchidan, bu fe'l shakli turk adabiy tilida ko'p ishlatilmaydi. Shuningdek, ise - esa to'liqsiz fe'li fe'lning murakkab shakllariga qo'shilganida, faqat shart mayliga xos ma'noni yuklaydi. Bu esa ayni fe'l shakllarini alohida mayl turi degan xulosaga kelish uchun yetarli emas. Fikrimizcha, bu fe'l shaklini shart modalligi deb tadqiq etish mumkin.

Demak, turk olimlarining qo'shma va qavatli qo'shma mayl xususidagi fikrlari olib borilgan tadqiqotda $\mathrm{o}^{6} \mathrm{z}$ isbotini topmadi. Xususan, turk tilshunoslari rus turkologlaridan farqli ravishda shart, keraklilik, buyruq, istak mayllarining zamon shakllari mavjudligini inkor etadilar. Rus, gruzin tilshunoslari esa qayd etilgan mayllarning idi to'liqsiz fe'li ishtirokida ishlatilishi muayyan maylning o'tgan zamon shaklini yuzaga keltiradi, degan xulosani bildirishadi.

Masalan, keraklilik mayli -meli affiksi orqali yasaladi. -meli va idi to 'liqsiz fe'lining qisqa yoki to'liq shaklidan yasalgan fe'l shakli keraklilik maylining o'tgan zamon shaklini yasaydi:

Vipip için çöllerde, tehlikede o insana eş olmaltydr(R.H.Karay).

Buning uchun cho 'llarda, taxlshada o'sha insonga umr yo'ldoshi bo'lishi kerak edi.

Quyidagi misolda esa shart maylining o'tgan zamon shakli deb ko'rsatiluvchi -se idi, sa edi formasi mavjud:

Oltauasak şeu ata Nasan Veu ve bep Azize'siz ve Saffet'siz tesadüf etseydik ve evlenseydik her halde çok kuvvetli bağa rağmen ben şiddetle bedbaht olacaktım (H.E.Adıvar).

Bo'lmagai narsa-ku, ammo Hasanbey bilan men Azizasiz va Saffetsiz tasodifan uchrashsak va turmush qursak edi, har holda o'rtamizdagi kuchli bog 'liqlikka qaramay mei tezda badbaxt bo 'lar edim.

\section{$O^{\prime}$ zbek va turk tillaridagi mayl mezonlari farqlanishi masalasi}

$\mathrm{O}^{\prime}$ zbek va turk tillaridagi xabar mayliga xos o'tgan zamon shakllarining boshqa turlari ham shu tarzda bir-biridan farqlanadi, ya'ni ma'lum bir qo'shimchalarga xos ustama modal ma'nolar ularning yagona invariant ma'no - o'tgan zamon doirasida bir xil emasligini ko'rsatadi.

Mayl mezonlarini belgilash sabablaridan biri zamon kategoriyasida ham modallik u yoxud bu darajada mavjudligini, xabar maylining vazifasi esa obyektiv reallikni tasvirlashda so'zlovchi nuqtai nazariga tayanishini, demakki, voqe'likni to' $\mathrm{g}^{\prime}$ 'ridan-to 'g'ri konstatatsiya etmasligini ko'rsatish edi. Bu fikr, bizningcha, ushbu tahlilda o'z isbotini topdi.

Bizningcha, mayl shaklini yasovchi formantlar subyektivlikni (so 'zlovchining nutqiga munosabatini) va obyektiv reallikni (o'tgan, hozirgi, kelasi zamondagi harakat-holat 
mavjudligining tasdig'i yoki inkorini) bildiradi. Modallik va zamonning mayl kategoriyasida birlashishiga sabab harakat va holat in'ikosidagi subyektivlik va obyektivlik o'rtasidagi o'zaro bog'liqlikdir. Obyektivlik darajasining ortishi, so'zsiz, subyektivlik hajmining kamayishiga olib keladi. Va aksincha, subyektivlik hajmining ortishi obyektivlik darajasining ozayishiga sabab bo'ladi. Agar obyektiv reallikni OR, modallikni (subyektiv reallikni) M deb olsak, bu hodisani quyidagicha sxemalashtirish mumkin(1-chizma):

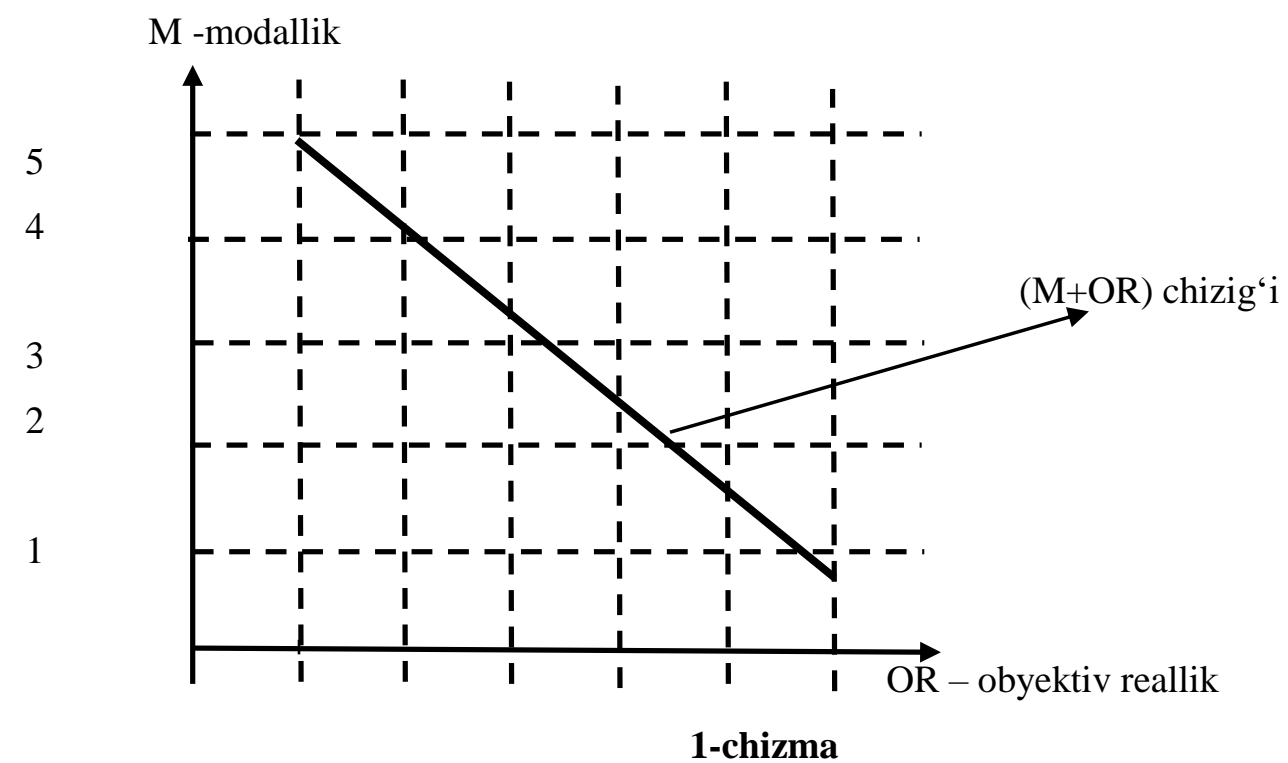

Ko'ramizki, bu hodisaning kulminasion nuqtasida modallik ham, obyektivlik ham nolga teng kelmaydi. Zero, harakat va holat subyektiv tasavvurlar qobig'ida aks etgani tufayli modalliksiz konstatatsiya amalga oshirilmaydi. Haqiqatdan ham, xabar mayli mohiyatan biroz farq qilsa ham, boisa mayllarga zid emas. Xabar maylidan boshqa mayllarning vazifasi modal munosabat bildirish, ayni vaqtda u voqe'likni ham qayd etadi. Xabar maylining birlamchi vazifasi harakat-holatni aks ettirish bilan xarakterlanadi. Ammo bu xabar maylida modallik yo'q, degani emas. Modallik birinchi o'rinda turmaydi, lekin mazmunning tarkibiga kirdi. Chunki xabar maylidagi fe'llardan anglashilgan ish-harakat ham subyektiv sharoit asosida yuzaga keladi. Bu o'z navbatida mayl va zamonni o'zaro aloqada o'rganishni talab etadi.

Zamon, asosan, uch ko'rinishga ega: o'tgan, hozirgi, kelasi. Mayl esa bevosita inson tafakkuriga bog'liq jarayon bo'lganligi uchun rang-barang bo'yoqlarda tovlanishi mumkin. Shuning uchun modallik obyektiv reallikka nisbatan ko'lamdor va boy. Shu sababli zamon sistemasi barcha mayllar tarkibida o'rganilishi kerak, degan fikrni ilgari surish mumkin.

Misollarga murojaat qilaylik:

- Oysha, men sen bilan bugun ochiqchasiga gaplashmoqchiman (P.Qodirov).

Bu gapda ishtirok etgan gaplashmoqchiman so'zi maqsad mayliga xos. So'zning ma'nosidan maqsad, xohish, istak anglashiladi. Shuningdek, so'zning mazmun tarkibida hozirgi-kelasi zamon tushunchasi bor. Ya'ni u bugun gaplashmoqchi, ertaga gaplashmoqchi yoki soat ikkida gaplashmoqchi, lekin kecha gaplashmoqchi emas. Turk tiliga ushbu gap quyidagicha o'giriladi:

\section{- Ayşe, bep bugüp senle açık konuşacağım.}

Ya'ni -moqchi formantining muqobili sifatida turk tilidagi -asak kelasi zamon affiksi ishlatiladi. Bu affiks bugün /bugun, yarın/ ertaga so'zlarini talab qilishi mumkin. 
-moqchi / -acak affiksi yordamida yasaluvchi fe'llarinnig nutq onidan avvalgi paytga oid voqea-hodisani ifodalashi uchun edi - idi to 'liqsiz fe'lidan foydalanish zarur:

$U$ avval Oyshaxon bilan yakkama-yakka gaplashmoqchi edi( $O$ '. Hoshimov).

Bir olau çıkarmanızdan korkuyordum, sırf size sevgi ve saygımdan bunu önleyecekim (R.H.Karay).

Bir falokatni boshlashiigizdan qo'rqayotgan edim, faqat sizga bo'lgan sevgim va hurmatim tufayli bunga mone'lik qilmoqchi edim.

Demak, gaplashmoqchi so'zida modallik obyektiv reallikdan ustun ( $\mathrm{M}>>\mathrm{OR}$ ), obyektiv reallik ham nolga teng emas( $M>>O R>>0)$. Ya'ni -moqchi affiksli fe'llarning asosiy xususiyati so'zlovchining modal munosabati, masalan, maqsadini ifoda etish bo'lsa-da, harakatning bajarilish vaqti ma'nosi ham anglashiladi.

Quyidagi gap tarkibida shart maylidagi fe'l to 'liqsiz fe'l bilan birikib qo'llangan:

Öule yapmasa, kisa bir flörtten sonra beraber yaşasak veya evlenseydik ötekileri gibi Nilgün'ü de unutur muydum? (R.H.Karay)

Shunday qilmasa, qisqa muddatlik flirtdan so 'ng birga yashasak yoki turmush qursak edi, boshqalari kabi Nilgunni ham unutarmidim?

Yaşasak veya evlenseydik /yashasak yoki turmush qursak edi so'zlari fe'ldan anglashilgan shartning nutq onidan oldin mavjud bo'lganligi, biroq amalga oshmaganligi ma'nosini bildiradi. Ya'ni shart maylidagi fe'l bildirgan ma'no to'liqsiz fe'lning ta'siri tufayli o'tmish foniga ko'chgan. Agar -ca, -se formantli fe'l to'liqsiz fe'l yordamisiz ishlatilsa, gapning umumiy mazmunidan ifodalangan shartning nutq oniga yoki kelasi zamonga oidligi ma'nosi kelib chiqadi:

Zeyno hanım gibi ava gelmesini tavsiye ederseniz nasil olur Saffet Veu! (H.E.Adıvar) Saffetbey!

Azizaga ham Zeyno xonim kabi ovga chiqishni tavsiya etsangiz qanday bo ladi,

Demak, bu holda modallik obyektiv reallikdan ustun(M $>>0 R>>0)$.

Keldi -geldi, kelgan, kelibman - gelmiş, kelib edi - geldiydi, kelar edi - gelirdi tipidagi so'zlar harakatning o'tgan zamonda bajarilganligining tasdig'ini ifodalaydi.

Masalan:

Shu payt darvozadan Sattor kirib keldi(O'.Hoshimov).

G'akat sabah olipsa Azize'nin yalvarmasına karşı yumuşadım ve o gün Göksu’ya gittik(H.E.Adlvar). ketdik.

Faqat tong otgach, Azizaning yolvorishlaridan yumshadim va o'sha kuni Ko 'ksuvga

Nil bir şeyler kararlastırmış(R.H.Karay).

Nil bir narsalarni rejalashtiribdi. Biz ota-onalarimizning ko'ngliga, xohishiga bo'ysunganmiz.

Yüzüne baktım, anladım: şakası yok. Nil bu müthiş kararı almış(R.H.Karay).

Yuziga qaradim, tushundim: hazil emas. Nsl bu mudhish qarorga kelgan.

Tashqi ko'rinishdan ajratib ko'rsatilgan fe'llarda modallik "0" (nol) ga tengdek ko'rinadi. Biroq o'tgan zamonda bajarilgan harakatni qayd etish bilan bir vaqtda keldi, yumuşadım - yumshadim, gittik - ketdik so'zlari yaqin o'tmishga oid aniq harakatni ifodalaydi. Kararlastırmış - rejalashtiribdi fe'li -di affiksli fe'lga nisbatan ilgari amalga 
oshirilgan ish-harakatning noaniqligini va so'zlovchi bu haqda boshqa manbalardan xabardor bo'lganligini bildiradi. bo'ysunganmiz, kararı almış — qarorga kelgan misolidagi -miş/ -gan shaklli fe'l o'tmishga oid harakatning nutq onida ham mavjudligini anglatadi. Keltirilgan misollardagi noaniqlik - aniqlik, eshitilganlik — guvohdek tasvirlash, yaqin o'tgan zamon uzoq o'tgan zamon, o'tmishga oid harakatning holatga o'tishi ma'nolaridan muayyanining borligi, aniq o'tgan zamon (keldi), uzoq o'tgan zamon (kelgan), o'tgan zamon hikoya fe'li (kelib edi), o'tgan zamon eshitilganlik fe'li (kelibman), o'tgan zamon davom fe'lidagi (kelar edi) modallik yuqoridagi xulosaning to'g'ri emasligini ko'rsatadi. Demak, xabar mayli voqe'likni to'g'ridan-to' $\mathrm{g}$ 'ri konstatatsiya qilsa ham, bu vazifa modalliksiz bajarilishi mumkin emas( OK $>\mathrm{M} \gg 0$ ).

Mayl va zamon formantlarini aynan shu asosda tadqiq qilish orqali bu muammoning munozarali bo'Igan ba'zi tomonlariga barham berish hamda mayl va zamon o'rtasidagi chegarani aniq belgilash mumkin, deb o'ylaymiz. Mayl va zamon kategoriyalarini hosil qiluvchi shakllarning soni va chegarasini belgilashdagi har xillikka chek qo'yishning vositasi bu ikki kategoriyani belgilovchi mezonlarni farqlashdir. Ayni mezonlar tarzida modallik va obyektiv reallik darajalarini ko'rsatish mumkin. Tavsiya qilinayotgan ushbu mezonlar xabar mayli bilan boshqa mayllar chegarasini aniq belgilashga yordam beradi. Chunki odatda, bir affiks yoki xabar maylining biron-bir zamon shakli yoki tamomila yangicha mayl deb ta'riflanadi. (Masalan, -ar edi ba'zi tadqiqotlarda xabar maylining o'tgan zamon davom fe'lini yasovchi qo'shimcha sifatida baholansa, ayrim ishlarda shartli mayl formanti, deb farqlanadi. Yoki -r/mas affiksli fe'llar ayrim manbalarda taxmin-gumon mayli deb, ba'zilarida kelasi zamon gumon fe'li deb ko'rsatiladi.)

\section{Xulosa}

Demak, mayl va zamon kategoriyalarining o'zaro munosabatini tadqiq etish borasida quyidagi xulosalarga kelindi:

Mayl va zamon biri ikkinchisi bilan uzviy bog'liq kategoriyalar bo 'lib, ular morfologik va semantik jihatdan qat'iy differensiatsiyalashmaganligi uchun zamon kategoriyasini shu kategoriyalarning o'zaro munosabati asosida tahlil qilish lozim. Shuningdek, bu ikki parallel jarayon doimiy uzviy aloqada ekanligini hisobga olmog'imiz darkor. Mayl mohiyati zamon mohiyatining negizida shakllanishi mumkin — bu mayl formalarini hosil qilishning usulidir. Ayni paytda zamon mayl negizida shakllanishi mumkin — bu zamon formalarining hosil qilish usullaridan biridir.

Mayl shaklini yasovchi formantlar subyektivlikni (so'zlovchining nutqiga munosabatini) va obyektiv reallikni (o'tgan, hozirgi, kelasi zamondagi xatti-harakatning, holatning tasdig'i yoki inkori)ni bildiradi. Mayl va zamon kategoriyalarini hosil qiluvchi shakllarning soni va chegarasini belgilashdagi har xillikka chek qo'yishning vositasi bu ikki kategoriyani belgilovchi mezonlarni farqlashdir. Ayni mezonlar tarzida modallik va obyektiv reallikni ko'rsatish mumkin.

Obyektivlik darajasining ortishi subyektivlik hajmining kamayishiga olib keladi. Subyektivlik hajmining ortishi, aksincha, obyektivlik darajasining kamayishiga sabab bo'ladi. $\mathrm{Bu}$ hodisaning kulminasion nuqtasida modallik va reallikning nolga teng kelishi mumkin emas. Chunki subyektivlikdan holi obyektivlik, obyektivlikdan tashqari subyektivlik yo'q.

Xabar mayliga xos o'tgan zamon shakllari voqe'likni qayd etishda so'zlovchining nuqtai nazariga, demakki, modal munosabatiga tayanadi, shu bois bu fe'l shakllari tarkibiga ustama modal ma'nolar yuklanadi. 


\section{Adabiyotlar}

BASKAKOV, N.A. (1966). Sistema spryajeniya ili izmeneniya slov po lisam v yazikax tyurkskoy gruppi, Issledovaniya sravnitelnoy grammatiki tyurkskix yazikov. Morfologiya. Ch.1. Moskva.

DENY, J. (1921). Grammaire De La language Turgue. Paris. drusk.

DENY, J. (1969). Rhilologiae Turcical Fundamenta. Wiesbaden, Wiesbaden satz und

DJANAShIA, N.N. (1981). Morfologiya tureskogo glagola. Tbilisi, Izd-vo Tbilis. GU.

DMITRIEV, N.K. (1960). Tureskiy yazik. Moskva: Izd-vo inos.lit-ri.

ERDEM, M. (2016). "Türkçede Fiiller ve Sınıflandırma Sorunları / Verbs and Issues of their Classifications in Turkish", TURKISh STUDIES -International Periodical for the Fall 2016, http://dx.doi.org/10.7827/TurkishStudies.10111, p. 185-200. ANKARA / TURKEY, www.turkishstudies.net.

ERGIN, M. (1993). Türk Dili Bilgisi. Istanbul: Vaurak basimevi.

G‘ULOMOV, A. G‘. (1964). Fe’l. Toshkent: Fan.

GENJAN, T. N. (1971). Dilbilgisi. Istanbul, Fen fekultesi basimevi. vostokoved.

GORDLEVSKIY, V. A. (1928). Grammatika tureskogo yazika. Moskva, Izd-vo ins-ta MGU.

GRUNINA, E.A. (1961). Istoricheskaya grammatika tureskogo yazika. Moskva, Izd-vo

HOJIEV, A. (1973). Fe'l. Toshkent: Fan.

IVANOV, S. N. (1977). Kurs tureskoy grammatiki: Grammaticheskie kategorii glagola. Leningrad, Izd-vo Deningrads. un-ta.

KONONOV, A. N. (1960). Grammatika sovremennogo uzbekskogo literaturnogo yazika. Moskva: Izd Vo AN SSSR. AN SSSR.

KONONOV, A.N. (1966). Grammatika tureskogo yazika. Moskva: Leningrad, Izd-vo

HATIBOĞLU, V. (1969). Dilbilgisi Terimleri Sözlüğü. Ankara: Türk Dili Kurumu.

HATIBOĞLU, V. (1974). Türkçenin Ekleri. Ankara: Türk Dili Kurumu.

RAHMATULLAEV, Sh. (1993). O'zbek va rus tillarini qiyoslash. Toshkent: Fan.

RESHETOV, V. V. (1961). Osnovi fonetiki i grammatiki uzbekskogo yazika. Tashkent: Srednyaya i visshaya shkola. vo AN SSSR.

SHERBAK, A. (1962). Grammatika starouzbekskogo yazika. Moskva: Leningrad: Izd-

SHOABDURAHMONOV, Sh.; ASQAROVA, M. (1980). Hozirgi o 'zbek adabiy tili. Toshkent: Fan.

SHUKUROV, Sh. (1976). O 'zbek tilida fe'l zamonlari taraqqiyoti. Toshkent: Fan.

SODIQOVA, M. (1976). Fe'l stilistikasi. Toshkent: Fan.

TANE, V. (1994). Türkçe Dil Bilgisi. İstanbul: Süret basım ve dağıtım A.S.

TOPARLI, R. (1991). Türk Dili. Erzurum: Fen Edebiyat Fakultesi Ofser Tesisleri. 
TURSUNOV, U., MUXTOROV, A., RAHMATULlAEV, Sh. (1992). Hozirgi o 'zbek adabiy tili. Toshkent: $\mathrm{O}^{\prime}$ zbekiston.

BANGIOGLU, T. (1990). Türkçenin Grammeri. Ankara: Türk tarih kurumu basım evi.

VIGUIER, I. (1790). Elements De La Langue Turgue. Sonstantinople.

WEIL, G. (1917). Grammatik Des Osmanischtürkischen Sprach. Verlin.

XO'JAEV, T. (1963). O‘zbek tilida fe’l va mayl, zamon va mayl masalalari. Trudi SamGU, № 124, 103. 